\title{
Minor actinides impact on basic safety parameters of medium-sized sodium-cooled fast reactor
}

Piotr Darnowski, Nikolaj Uzunow

\begin{abstract}
An analysis of the influence of addition of minor actinides (MA) to the fast reactor fuel on the most important safety characteristics was performed. A special emphasis was given to the total control rods worth in order to describe qualitatively and quantitatively its change with MA content. All computations were performed with a homogeneous assembly model of modified BN-600 sodium-cooled fast reactor core with 0,3 and $6 \%$ of MA. A model was prepared for the Monte Carlo neutron transport code MCNP5 for fresh fuel in the beginning-of-life (BOL) state. Additionally, some other parameters, such as Doppler constant, sodium void reactivity, delayed neutron fraction, neutron fluxes and neutron spectra distribution, were computed and their change with MA content was investigated. Study indicates that the total control rods worth (CRW) decreases with increasing MA inventory in the fuel and confirms that the addition of MA has a negative effect on the delayed neutron fraction.
\end{abstract}

Key words: fast reactor safety $\bullet$ MCNP5 $\bullet$ sodium-cooled fast reactor $\bullet$ nuclear waste transmutation

P. Darnowski ${ }^{\bowtie}, \mathrm{N}$. Uzunow Institute of Heat Engineering, Warsaw University of Technology, 21/25 Nowowiejska Str., 00-665, Warsaw, Poland, Tel./Fax: +48 22234 5297,

E-mail: piotr.darnowski@itc.pw.edu.pl

Received: 25 April 2014

Accepted: 23 January 2015

\section{Introduction}

The fast spectrum reactor technology has a development history almost as long as the nuclear power. The idea appeared in the late forties of the twentieth century with the construction of the Clementine core and it was extensively developed for over fifty years [1]. The promise of almost inexhaustible energy source was very tempting, and the extensive R\&D funding for several years can only be compared to fusion reactors technology. Nevertheless, the fast breeder reactors (FBR) were never introduced on a large scale. However, it is remarkable that the technology became very mature [2-4]. The maturity of sodium-cooled fast reactor technology was proven by plenty of installations, especially by BN-600, BN-350, and Phénix reactors. These installations worked for several decades, produced large quantities of energy, and were very safe even as they were prototypical ones $[2,4,5]$. The golden era of fast reactor development ended in the late eighties with the end of arms race and the Chernobyl accident, when global political and social reliance in nuclear energy almost disappeared [3]. Fortunately, the development of fast reactors never stopped in some countries - especially in Russia, China and India. An important change in the direction and thinking about this technology was the creation of the International Generation IV Forum, which started to promote the idea of wide implementation of fast reactors [2, 6, 7]. New concepts for this technol- 
ogy were introduced, and the most promising is the application of fast neutron spectrum to burn and transmute nuclear waste together with innovative reprocessing technologies - the idea of partitioning and transmutation (P\&T) $[7,8]$.

For many years, the fast reactors were perceived as nuclear fuel breeders, or rather transuranium nuclides (and radiotoxicity) production facilities. Modern burner or converter fast reactors are designed to effectively utilize nuclear fuel and reduce total net radiotoxicity and finally to close the whole fuel cycle [2]. In the framework of the P\&T, mainly transuranium (plutonium and minor actinides) nuclides are considered to be burned.

The fission products (FP) are responsible for most of the radiotoxicity of spent nuclear fuel during the first few hundred years after the end of irradiation [8]. Fortunately, most of the FPs are rather short-lived nuclides. However, there are also some very long-lived fission products. Nevertheless, their impact on the overall radiotoxicity is practically negligible in long terms [9]. Burning of FPs in burner reactors is considered as impractical in current perspective [2]. From the technical point of view, the today technology can guarantee safe storage of spent fuel with very high credibility for hundreds of years, and it is rather the problem of very-long-term radiotoxicity that should be resolved. Additionally, the decrease of radiotoxicity and decay heat should lead to significant improvement of final disposal facilities storage capacity by effective reduction of the volume necessary to storage a unitary mass of nuclear waste [10].

A very promising approach to transmutation are the accelerator-driven systems (ADS) - subcritical reactors dedicated to transmutation, which have very little obstructions to the MA content in the fuel [11]. Nevertheless, the ADS have some technical limitations: practically, they cannot have more than $1000 \mathrm{MW}_{\mathrm{th}}$, and in consequence they cannot be large power production installations [12]. On the contrary, the critical fast reactors have limitations on

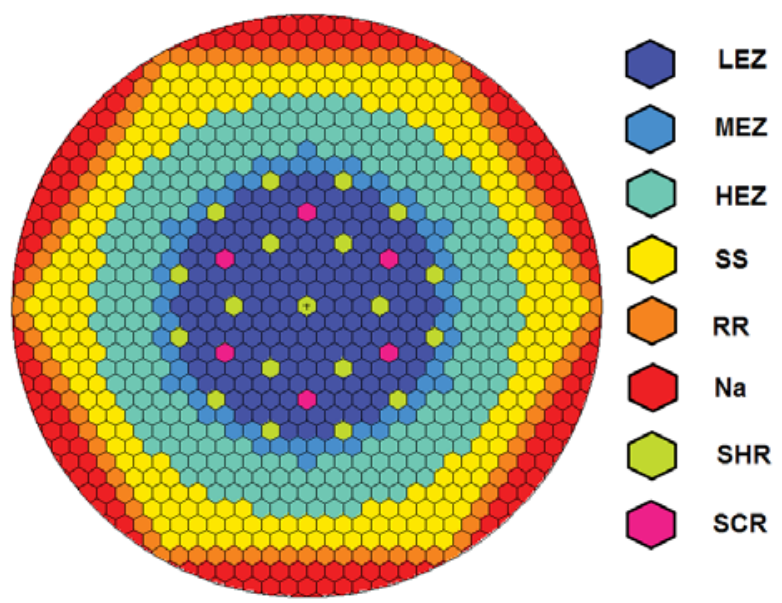

Fig. 1. Horizontal cross-section of the core. SS - steel radial reflector; $\mathrm{RR}$ - stainless steel radial reflector; $\mathrm{Na}$ - sodium; LEZ - low enrichment zone; MEZ - medium enrichment zone; HEZ - high enrichment zone; SHR and SCR are control assemblies. the MA content (no more than 5-6\%) due to safety concerns, but they can have relatively large power [12]. Additionally, in the case of critical fast reactors, it should be relatively simple to redesign them, if it will be necessary, to become breeder reactors and produce fissile materials - new fuel.

Special concern should be taken to the minor actinides, which are responsible for almost all spent fuel long-term radiotoxicity. Fast neutron spectrum is very suitable to transmute MA into short-lived or stable nuclides, especially due to higher relative fission-to-capture cross-sections than in the thermal spectrum. The addition of MA into the fuel with reduction of the fertile inventory makes net destruction of those nuclides possible. The crucial minor actinide is americium, as it has the largest contribution to radiotoxicity in the period of 1000 to 100000 years after irradiation. The reduction of Am inventory is believed to be the most important purpose of transmutation [7-12].

\section{Core model}

In this work, a model of reactor core with homogenized assemblies was created for the Monte Carlo neutron transport code MCNP5 [13]. For simplicity and in order to significantly reduce the computational effort, every hexagonal assembly was divided into few axial levels, and the material was homogenized respectively (Figs. 1, 2). The homogenization was based on the core geometrical parameters, and on the typical nuclear density computation methodology, described in $[2,14,15]$. Worth to mention that the homogenization done in this work is fundamentally different than the standard homogenization used in neutron diffusion methods.

The homogenization approach cannot be applied with Monte Carlo when the mean free path

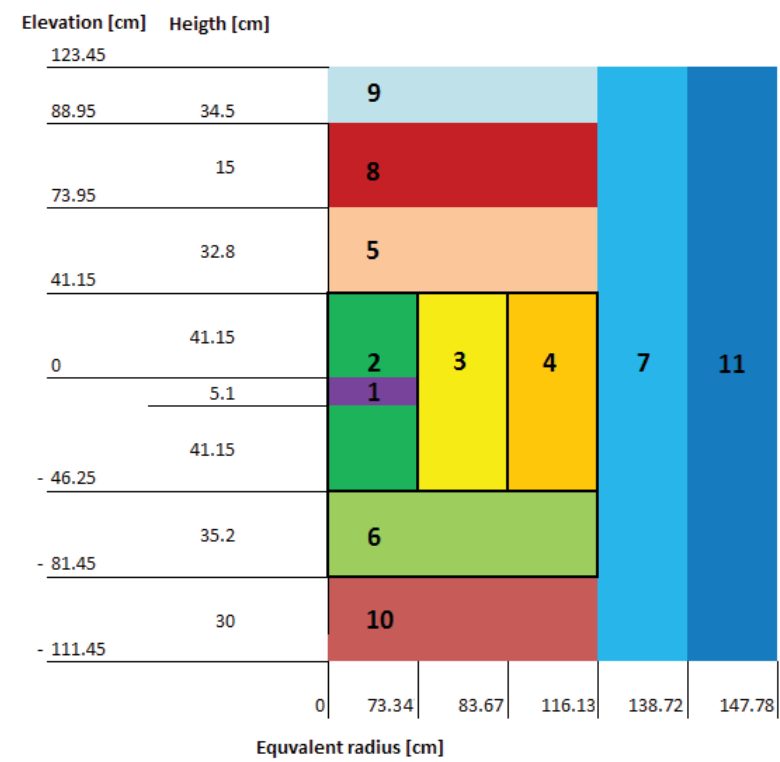

Fig. 2. Axial and radial zones of the model. 1 - IBZ internal breeding zone; 2 - LEZ; 3 - MEZ; 4 - HEZ; 5 - upper sodium plenum; 6 - lower axial blanket; 7 - steel radial shield; 8 - upper boron shield; 9 - upper reflector; 10 lower reflector; 11 - radial reflector. 
of neutrons is comparable to the fuel rods lattice pitch, what is the case for typical thermal reactors. In the case of fast neutrons, the mean free path is larger than the elementary lattice pitch length. In some core designs it could be even higher than the characteristic dimension of sub-assembly (SA - fuel assembly), so from the neutrons point of view those are quasi-homogeneous.

As the thermal neutrons are negligible in fast reactors' neutron spectrum, such a simplification could lead to acceptable physical results [16-18].

Table 1. Principal geometric parameters of BN600 model

\begin{tabular}{lc}
\hline Parameter & Value \\
\hline Subassembly (SA) pitch & $9.902 \mathrm{~cm}$ \\
Pitch-to-diameter ratio (P/D) & 1.1522 \\
Distance between SA walls & $0.24 \mathrm{~cm}$ \\
SA canister thickness & $0.20 \mathrm{~cm}$ \\
Fuel pin outer diameter & $0.69 \mathrm{~cm}$ \\
Cladding thickness & $0.04 \mathrm{~cm}$ \\
Blanket pin outer diameter & $1.40 \mathrm{~cm}$ \\
Blanket pin cladding thickness & $0.04 \mathrm{~cm}$ \\
SCR/SHR rod outer diameter & $2.3 \mathrm{~cm}$ \\
SCR/SHR outer canister thickness & $0.2 \mathrm{~cm}$ \\
SCR/SHR inner canister thickness & $0.1 \mathrm{~cm}$ \\
SCR/SHR cladding thickness & $0.04 \mathrm{~cm}$ \\
\hline
\end{tabular}

Table 2. Atomic fraction of $\mathrm{Pu}, \mathrm{U}$ and MA isotopes in different core zones. Cases: \# 1 for 0\% MA, \#2 for 3\% MA, and \#3 for $6 \%$ of MA. AB - axial blanket

\begin{tabular}{|c|c|c|c|}
\hline & $\begin{array}{c}\mathrm{Pu} \\
\text { [at.] }\end{array}$ & $\begin{array}{c}\mathrm{U} \\
\text { [at.] }\end{array}$ & $\begin{array}{l}\text { MA } \\
\text { [at.] }\end{array}$ \\
\hline \multicolumn{4}{|c|}{$\# 1$} \\
\hline LEZ & 0.20 & 0.80 & 0.0 \\
\hline MEZ & 0.21 & 0.79 & 0.0 \\
\hline HEZ & 0.22 & 0.78 & 0.0 \\
\hline IBZ & 0.0 & 1.0 & 0.0 \\
\hline $\mathrm{AB}$ & 0.0 & 1.0 & 0.0 \\
\hline \multicolumn{4}{|c|}{$\# 2$} \\
\hline LEZ & 0.20 & 0.77 & 0.03 \\
\hline MEZ & 0.21 & 0.76 & 0.03 \\
\hline HEZ & 0.22 & 0.75 & 0.03 \\
\hline IBZ & 0.0 & 1.0 & 0.0 \\
\hline$A B$ & 0.0 & 1.0 & 0.0 \\
\hline \multicolumn{4}{|c|}{ \#3 } \\
\hline LEZ & 0.20 & 0.74 & 0.06 \\
\hline MEZ & 0.21 & 0.73 & 0.06 \\
\hline HEZ & 0.22 & 0.72 & 0.06 \\
\hline IBZ & 0.0 & 1.0 & 0.0 \\
\hline $\mathrm{AB}$ & 0.0 & 1.0 & 0.0 \\
\hline
\end{tabular}

Table 3. Isotopic vectors for $\mathrm{U}, \mathrm{Pu}$ and MA
A comparison of full core model results with homogenized model results and experimental data from the JOYO reactor shows that this approach can be acceptable [18]. Nevertheless, the analysis of control rods effects requires special attention.

The reactor core used in this work was developed on the basis of modified BN-600 core with added minor actinides, described in the report of Rienski et al. [7] and [19, 20]. In our model (Fig. 1), some of the less important zones, such as plugs and cones, were merged with blankets or reflectors to simplify the problem. Furthermore, all structural elements were composed of austenitic steel SS316, which is representative for fast reactors [2]. In the case of real BN-600, the structural elements are made of ChS-68 alloy [21], but such a change should have rather a small influence on the results. All relevant geometrical data for the reactor was taken from [5, 22], and the most important dimensions are listed in Table 1. The core is divided into three main regions (Fig. 1): LEZ with low-enriched fuel, MEZ with medium enrichment, and outer core region HEZ with high enrichment. The core is coated with a steel radial shield (SS) and a radial reflector (RR) (Fig. 1). Axially, above the core there is an upper sodium plenum, upper boron shield and upper reflector (Fig. 2). Lower axial blanket $(\mathrm{AB})$ and lower reflector are located below the core. Inside the inner core LEZ region there is internal breeding zone (IBZ) dedicated to reduce sodium void effect [22] (Fig. 2). The remaining part of the system is filled with a sodium coolant. The relevant core dimensions are plotted in Fig. 2.

Minor actinides were added to the main three core regions with atomic fractions presented in Table 2. The isotope vector used to compose fuel mixture is shown in Table 3 . The uranium isotope vector corresponds to a PWR depleted uranium after 15 years of cooling [23]. The plutonium and MA vectors are based on those reported in [15]. A fuel smeared density of $87 \%$ of the theoretical density, and a substechiometric ratio of $\mathrm{O} / \mathrm{M}=1.98$ were used [2].

All control rods are located inside the LEZ region (Fig. 1) and in withdrawn state they are parked $1.85 \mathrm{~cm}$ above the core. The bottom plane of control rods in case of full insertion is $0.75 \mathrm{~cm}$ below the core (Fig. 2). In the model, there are nineteen shim control rods (SHR) and six scram control rods (SCR). All control rods are composed of boron carbide $\left(\mathrm{B}_{4} \mathrm{C}\right)$. The boron inventory for SHR is 20 at. $\%{ }_{5}^{10} \mathrm{~B}$ with remaining ${ }_{5}^{11} \mathrm{~B}$ (natural boron mixture). Differently, SCRs are made of enriched boron with 80 at. $\%{ }_{5}^{10} \mathrm{~B}$ and 20 at. $\%{ }_{5}^{11} \mathrm{~B}$ [5].

\begin{tabular}{lccccc}
\hline \multicolumn{2}{c}{ Plutonium $(\mathrm{Pu})$} & \multicolumn{2}{c}{ Uranium $(\mathrm{U})$} & \multicolumn{2}{c}{ Minor actinides (MA) } \\
\hline \multicolumn{1}{c}{ Isotope } & [at.] & Isotope & [at.] & Isotope & [at.] \\
\hline Pu-238 & 5.5 & $\mathrm{U}-234$ & 0.0031 & $\mathrm{~Np}-237$ & 36.9001 \\
$\mathrm{Pu}-239$ & 61.0 & $\mathrm{U}-235$ & 0.4091 & Am-241 & 53.2199 \\
$\mathrm{Pu}-240$ & 32.0 & $\mathrm{U}-236$ & 0.0101 & Am-243 & 8.2303 \\
$\mathrm{Pu}-241$ & 1.0 & $\mathrm{U}-238$ & 99.5777 & $\mathrm{Cm}-244$ & 1.3938 \\
$\mathrm{Pu}-242$ & 0.5 & - & - & $\mathrm{Cm}-245$ & 0.1166 \\
- & - & - & - & $\mathrm{Cm}-246$ & 0.1393 \\
\hline
\end{tabular}


A core thermal power of $1470 \mathrm{MW}_{\text {th }}$ was determined, and it was assumed that each fission of a heavy nucleus produces $200 \mathrm{MeV}[2,5]$.

\section{Methodology}

\section{Neutron flux}

The neutron energy spectrum was averaged over the whole core and normalized to a total averaged flux. A method of obtaining such a distribution in MCNP5 is described by Nifenecker et al. [11].

The spatial distribution of neutron flux in the core centre was computed using the method presented by Ravnik and Snoj [24]. MCNP tallies card FMESH was utilized, and a special mesh for neutron flux sampling created [13]. The code provides only a neutron flux normalized to one fission event, so it is necessary to adjust it to the reactor power [24]:

$$
\Phi=\frac{P \cdot v}{w_{f} \cdot 1.6022 \cdot 10^{-13}} \cdot \frac{1}{k_{e f f}} \phi_{\mathrm{F} 4}
$$

where: $P$ - reactor power $[\mathrm{W}] ; \Phi$ - neutron flux $\left[\mathrm{n} /\left(\mathrm{cm}^{2} \cdot \mathrm{s}\right)\right] ; v$ - average number of neutrons produced per fission event [n/fission]; $k_{\text {eff }}$ - effective multiplication factor $[-] ; w_{f}-$ average energy produced per fission [MeV/fission]; $1.6022 \cdot 10^{-13}$ is a scaling factor [J/MeV]; and $\phi_{\mathrm{F} 4}-$ neutron flux tally obtained with FMESH or F4 MCNP card. The radial flux was sampled in the volume limited by two planes located axially between $-5 \mathrm{~cm}$ and $+5 \mathrm{~cm}$ (Fig. 2).

\section{Delayed neutron fraction}

The delayed neutron fraction $(\beta)$ and effective delayed neutron fraction $\left(\beta_{\text {eff }}\right)$ were computed based on a method suitable for MCNP and described by Westlen [7] and Michalek et al. [25]:

$$
\begin{aligned}
& \beta=1-\frac{k_{3}}{k_{2}} \\
& \beta_{\text {eff }}=1-\frac{k_{3}}{k_{1}}
\end{aligned}
$$

where $k_{1}, k_{2}$ and $k_{3}$ are effective multiplication factors obtained in MCNP computations (with PHYS card modified). They are defined as follows:

$$
\begin{aligned}
& k_{1}=k_{p}+k_{d_{e f f}} \\
& k_{2}=k_{p}+k_{d} \\
& k_{3}=k_{p}
\end{aligned}
$$

In this case, $k_{p}$ is the part of $k_{\text {eff }}$ generated by prompt neutrons, $k_{\text {deff }}$ is the part of $k_{\text {eff }}$ generated by delayed neutrons, and $k_{d}$ is the part of $k_{e f f}$, which delayed neutrons would create if they have had the same spatial and energy distribution as prompt neutrons. TOTNU card was applied to obtain those multiplication factors $[7,13,25]$.

\section{Control rod worth}

Control rods worth (CRW) was obtained by computation of the effective multiplication factor for a core with control assemblies $\left(k_{2}\right)$ withdrawn (both SCR and SHR), and for a core with all control assemblies parked in their bottom position $\left(k_{1}\right)$. In order to estimate control rods worth, the following formula was applied [18]:

$$
\mathrm{CRW}=\frac{k_{1}-k_{2}}{k_{1} k_{2}}
$$

The standard deviation of CRW is given by the error propagation formula:

$$
\Delta \mathrm{CRW}=\left(\frac{\Delta k_{1}}{k_{1}^{2}}+\frac{\Delta k_{2}}{k_{2}^{2}}\right)
$$

In order to express CRW in relative reactivity units, Eq. (4) should be divided by the effective delayed neutron fraction $\left(\beta_{\text {eff }}\right)$, which normalizes CRW and expresses it in terms of dollars [\$]. The error propagation formula should be used again to compute the relative $\Delta$ CRW (Eq. (5)), because $\beta_{\text {eff }}$ is characterized by an additional standard deviation.

\section{Doppler feedback}

At normal core operation state, the fuel isotopes are assumed to be at an uniform temperature of $1200 \mathrm{~K}$. All remaining structure, absorbers and coolant are at $600 \mathrm{~K}$. Default MCNP5 ENDFVII cross-section libraries were used in those calculations.

In order to evaluate the Doppler reactivity constant (KD), the fuel isotopes temperature was changed to $2500 \mathrm{~K}$ to simulate core heat-up. KD is given by the following relation $[18,21]$ :

$$
\mathrm{KD}=\frac{k_{2}-k_{1}}{k_{2} k_{1}} \cdot \frac{1}{\ln \left(T_{2} / T_{1}\right)}
$$

where $k_{1}, T_{1}$ are the $k$-factor and temperature for the initial core state with control rods withdrawn. Differently, $k_{2}, T_{2}$ are effective multiplication factor and temperature for heated core. The standard deviation is given by Eq. (7) [18]:

$$
\Delta \mathrm{KD}=\left(\frac{\Delta k_{1}}{k_{1}^{2}}+\frac{\Delta k_{2}}{k_{2}^{2}}\right) \cdot \frac{1}{\ln \left(T_{2} / T_{1}\right)}
$$

The Doppler reactivity coefficient $\alpha_{D}$ can be computed using:

$$
\alpha_{\mathrm{D}}=\frac{\mathrm{KD}}{T_{2}}
$$

\section{Sodium void reactivity}

The sodium void reactivity was estimated for a core with sodium nuclear and mass density lowered by a factor of one thousand (assumed for simplicity) and for core with fully withdrawn control rods. This approach was applied to LEZ, IBZ, MEZ and HEZ regions. The sodium void reactivity (SVR) can be estimated by the following relation [18]: 


$$
\operatorname{SVR}=\frac{k_{1}-k_{2}}{k_{1} k_{2}}
$$

where $k_{1}$ is the effective multiplication factor for core in normal state and $k_{2}$ was obtained for voided core. The standard deviation can be determined using the error propagation formula:

$$
\Delta \mathrm{SVR}=\left(\frac{\Delta k_{1}}{k_{1}^{2}}+\frac{\Delta k_{2}}{k_{2}^{2}}\right)
$$

It is worth to mention that the larger negative value of the sodium void reactivity (SVR), physically leads to more positive insertion of the reactivity. It is opposite effect than for other reactivity effects and it could lead to confusion.

\section{Results}

All the performed computations were based on the Evaluated Nuclear Data File 7.0 (ENDF7), default nuclear libraries for MCNP5 [13]. For each computation, 500 active cycles (with MCNP criticality mode) were performed with 30 initial cycles and 30000 neutrons per active cycle $[13,14]$.

This gives fifteen million neutrons per one case. The standard deviations for the multiplication factor $\left(k_{\text {eff }}\right)$ were in the range of $0.00012-0.00013$.

Radial distribution of total neutron flux is presented in Fig. 3. The whole core spatially weighted

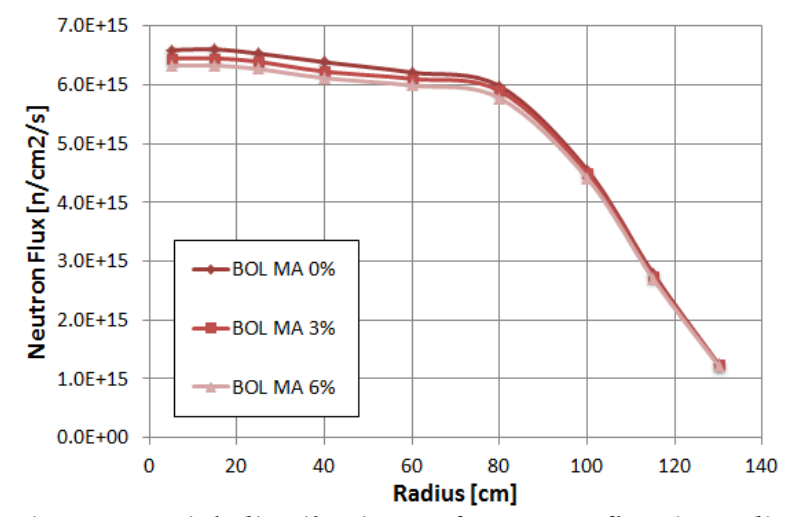

Fig. 3. Spatial distributions of neutron flux in radial direction for different MA content. The control rods are withdrawn.

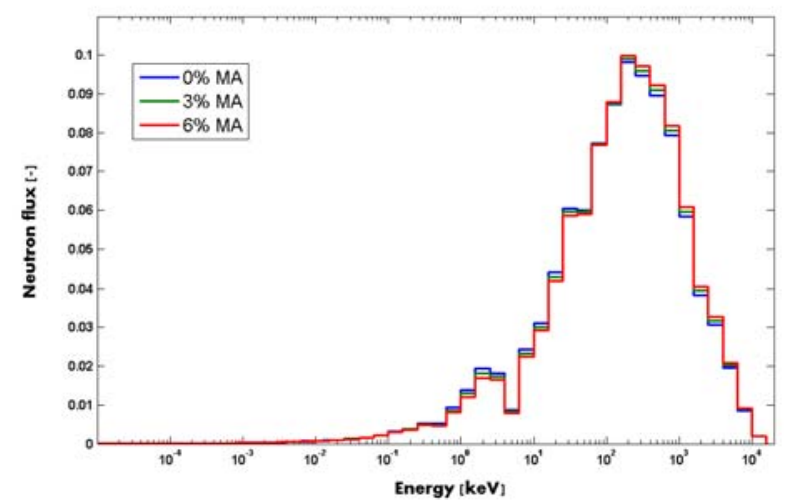

Fig. 4. Spectral distribution of normalized neutron flux for different MA content. Spectra sampled over core volume and control rods outside the core.

neutron energy spectra are shown in Fig. 4. Both are for three different minor actinides content in the fuel.

The average numbers of neutrons produced per fission $(v)$ were 2.922, 2.932 and 2.943 for MA content 0,3 and $6 \%$, respectively. The average neutron energies causing fission were 843,896 and $944 \mathrm{keV}$ for 0,3 and $6 \%$, respectively.

The reactor core reactivity for fully withdrawn and fully inserted control rods as a function of MA content are shown in Fig. 5 with detailed data and standard deviations presented in Table 4 . The fuel Doppler constant and sodium void reactivity with deviations are graphed in Figs. 6, 7 and data presented in Table 4.

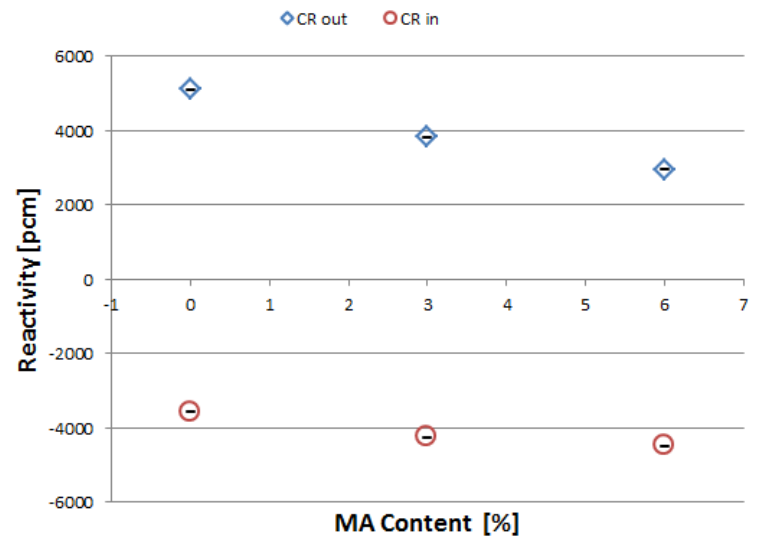

Fig. 5. Reactivity for CR withdrawn (diamonds) and reactivity for CR inserted (circles). Black dashes are standard deviations.

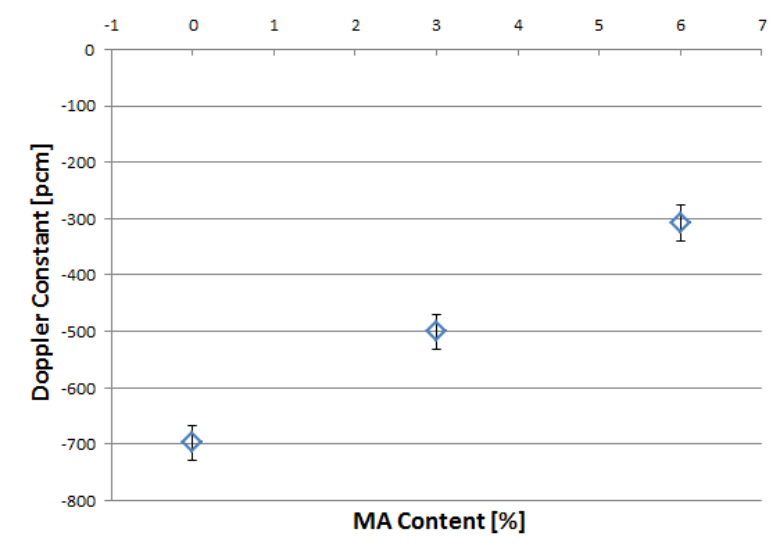

Fig. 6. Fuel Doppler constant (KD).

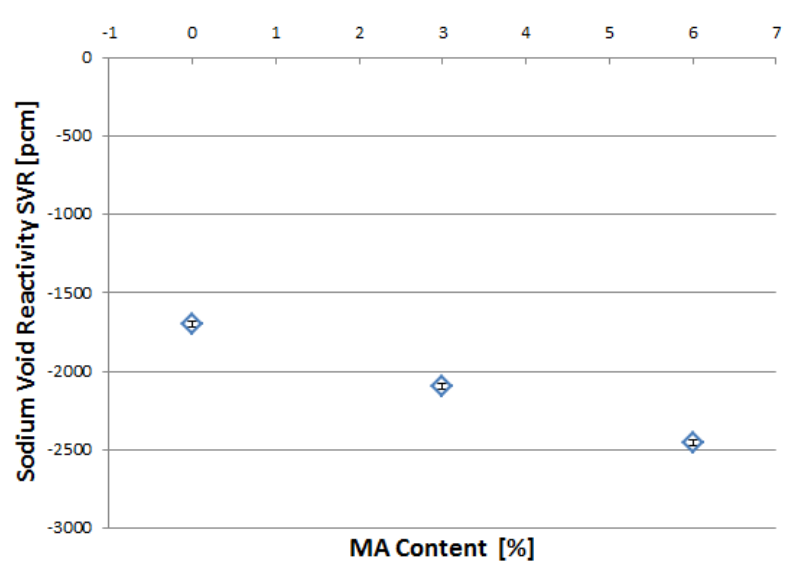

Fig. 7. Sodium void reactivity (SVR) in absolute values. 
Table 4. Results of important safety parameters for different MA content with standard deviations. $\rho_{\text {out }}-$ reactivity of the core in normal state with all control rods withdrawn, $\rho_{\text {in }}$ - core reactivity with all control rods fully inserted

\begin{tabular}{lccc}
\hline \multicolumn{1}{c}{ MA } & $0 \%$ & $3 \%$ & $6 \%$ \\
\hline$\rho_{\text {out }}[\mathrm{pcm}]$ & $5105 \pm 12$ & $3824 \pm 11$ & $2962 \pm 12$ \\
$\rho_{\text {in }}[\mathrm{pcm}]$ & $-3578 \pm 14$ & $-4247 \pm 13$ & $-4487 \pm 13$ \\
$\beta[\mathrm{pcm}]$ & $376 \pm 17$ & $353 \pm 17$ & $339 \pm 16$ \\
$\beta_{\text {eff }}[\mathrm{pcm}]$ & $307 \pm 17$ & $295 \pm 16$ & $287 \pm 17$ \\
CRW [pcm] & $8684 \pm 26$ & $8070 \pm 24$ & $7448 \pm 25$ \\
CRW [\$] & $28.33 \pm 1.61$ & $27.34 \pm 1.51$ & $25.93 \pm 1.55$ \\
SVR [pcm] & $-1698 \pm 22$ & $-2096 \pm 23$ & $-2456 \pm 24$ \\
SVR [\$] & $-5.54 \pm 0.32$ & $-7.10 \pm 0.40$ & $-8.55 \pm 0.51$ \\
KD [pcm] & $-697 \pm 31$ & $-501 \pm 30$ & $-309 \pm 32$ \\
KD $[\$]$ & $-2.27 \pm 0.11$ & $-1.70 \pm 0.09$ & $-1.07 \pm 0.07$ \\
$\alpha_{\mathrm{D}}[\mathrm{pcm} / \mathrm{K}]$ & $-0.2788 \pm 0.0123$ & $-0.2004 \pm 0.0121$ & $-0.1235 \pm 0.0129$ \\
\hline
\end{tabular}

The negative SVR coefficient means insertion of positive reactivity into the core. On the contrary, negative $\mathrm{KD}$ is equivalent with insertion of negative reactivity. The delayed neutron fractions and effective delayed neutron fractions are plotted in Fig. 8 and summarized in Table 4.

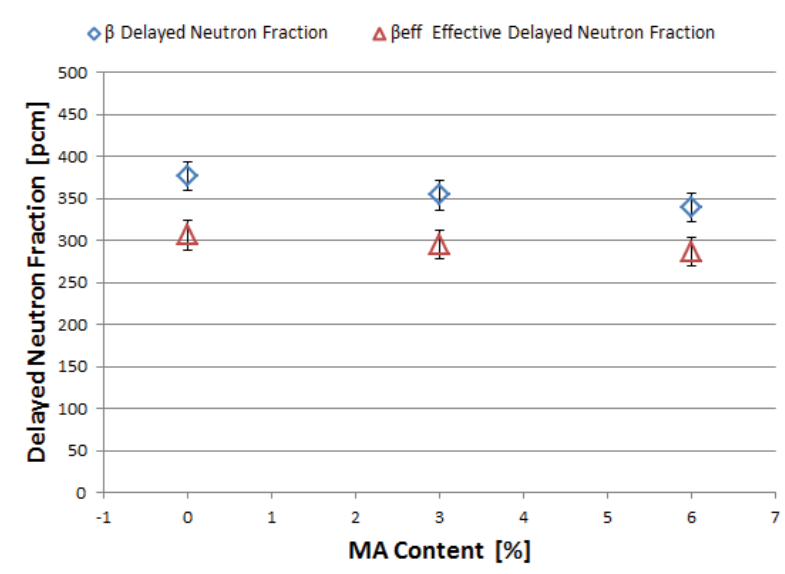

Fig. 8. Delayed neutron fraction $(\beta)$ and effective delayed neutron fraction $\left(\beta_{\text {eff }}\right)$.

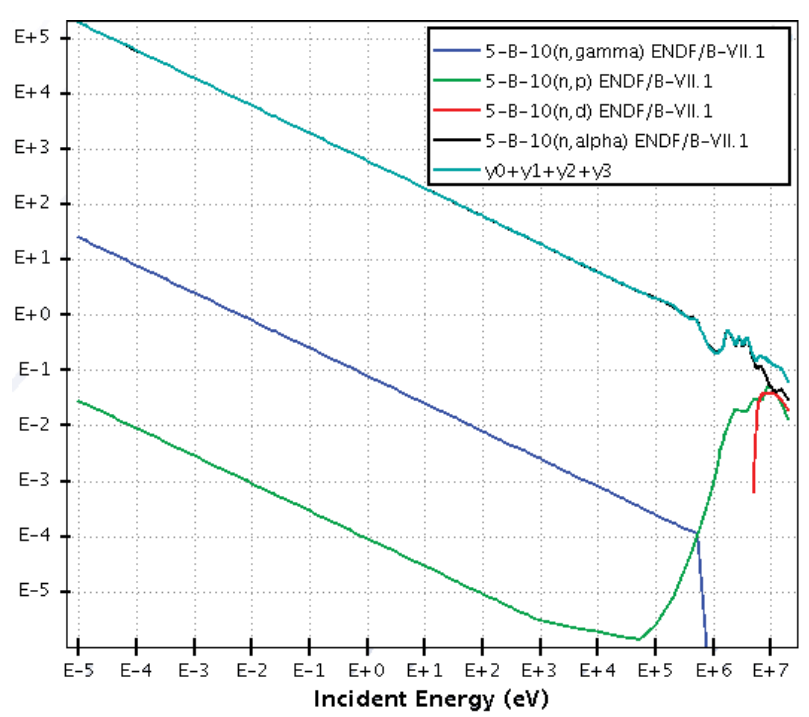

Fig. 9. Boron-10 capture cross-sections for different reaction mechanisms presented as a function of incident neutron energy. Curve labelled as $y 0+y 1+y 2+y 3$ is total capture cross-section [26].

\section{Discussion}

Figure 5 shows that the control rods absolute worth decrease with increasing content of MA in the fuel. It could be explained by examination of neutronic characteristics of the core. This problem will be investigated in the following paragraphs.

First of all, the neutron spectrum becomes harder with addition of MA (Fig. 4), due to increasing relative amount of prompt neutrons generated per fission for MA and less delayed neutrons produced by MA's fission products.

The principal absorbing isotope in the control rods is ${ }_{5}^{10} \mathrm{~B}$ and its neutron capture cross-sections for main mechanisms are plotted in Fig. 9. It is apparent that with increasing neutron incident energy (harder spectrum), total capture cross-section decreases.

The neutron capture reaction rate in control rods can be simply related to the cross-section and flux by the following relation [27]:

$$
R_{c}=\int N_{c} \sigma_{c}(E) \phi(E) d E
$$

Formulas (10) and (11) are spatially averaged. Consequently, harder neutron spectrum on the average should cause lower neutron capture reaction rates and effectively reduces worth of control rods (Table 4). Moreover, all the control rods are introduced into the LEZ core region, where the neutron flux is lowered for higher MA content (Fig. 3) - it is a spatial effect and Eq. (10) does not take it directly into account. Decreased flux should additionally influence in a negative manner the capture rate in

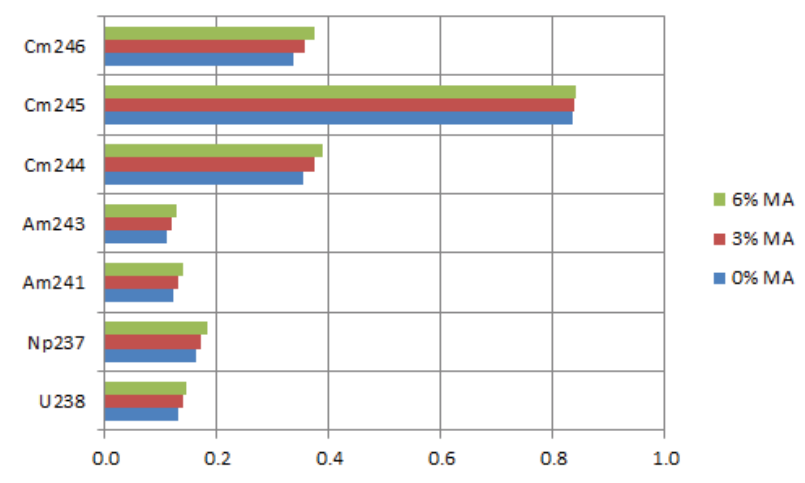

Fig. 10. Neutron spectrum weighted fission probability $\left\{\alpha^{-1}=\left[\sigma_{f} /\left(\sigma_{c}+\sigma_{f}\right)\right]=\left(\sigma_{f} / \sigma_{a}\right)\right\}$ of MA for LEZ region. 
Table 5. Capture and fission cross-sections for most abundant isotopes in fuel vector (Table 1). Values averaged for typical sodium-cooled reactor fast spectrum (based on [2])

\begin{tabular}{lccc}
\hline \multicolumn{1}{c}{ Nuclide } & $\bar{\sigma}_{f}[\mathrm{~b}]$ & $\bar{\sigma}_{c}[\mathrm{~b}]$ & $\bar{\sigma}_{f} / \bar{\sigma}_{c}$ \\
\hline${ }_{94}^{238} \mathrm{Pu}$ & 1.10 & 0.58 & 1.90 \\
${ }^{239}{ }_{94} \mathrm{Pu}$ & 1.86 & 0.56 & 3.32 \\
${ }_{240}^{240} \mathrm{Pu}$ & 0.36 & 0.57 & 0.63 \\
${ }_{94}^{238} \mathrm{U}$ & 0.04 & 0.30 & 0.13 \\
${ }_{92}^{237} \mathrm{~N}$ & 0.32 & 1.70 & 0.19 \\
${ }_{93}^{241} \mathrm{~Np}$ & 0.27 & 2.00 & 0.14 \\
${ }_{95}^{243} \mathrm{Am}$ & 0.21 & 1.80 & 0.12 \\
\hline
\end{tabular}

the poison material. The boron nuclear density was constant for the three analyzed cases.

Reactor power is produced according to neutron production by fission reactions [27]:

$$
R_{f}=\int N_{f} \sigma_{f}(E) \phi(E) d E
$$

The reactor overall power is the same for all the analyzed cases $\left(1470 \mathrm{MW}_{\mathrm{th}}\right)$, but the total neutron flux decreases with increasing MA content (Fig. 3). In order to maintain the same fission reaction rate, the fission nuclei density, microscopic fission cross-sections or neutron spectra should change (Eq. (11)). Particularly all those parameters increase in analyzed cores with increasing MA content.

In the analyzed fuel, the depleted uranium was gradually substituted with minor actinides. As a consequence, the addition of MAs causes a slight increase of the fissile material nuclear density (Table 2). What is more important, most of the minor actinides have higher fission probabilities and cross-sections in comparison to uranium (Fig. 10 and Table 5). This effect is stronger for higher MA inventory - due to harder neutron spectrum (Fig. 4 and Fig. 10). An effectively smaller neutron flux is necessary to perform the same amount of nuclear fissions and obtain the same power level.

What is remarkable, even with higher fissile material density, higher fission cross-sections and harder spectrum, less reactivity is produced $\left(\rho_{\text {out }}\right)$ in Table 4. The overall amount of nuclear reactions in added MA is much higher than in the substituted uranium for both capture and fission reactions, mainly due to low uranium cross-sections (Table 5) [2].

The total number of reactions should be much greater than for uranium, but the most abundant MA isotopes have low fission probability, hence more captures than fissions occur (Table 5 and Fig. 10). In fuel without MA, due to the low uranium cross-section, there is no significant competition for plutonium isotopes in absorption phenomena. The plutonium isotopes used in the fuel (Table 3) are mainly ${ }_{239}^{239} \mathrm{Pu}$ and ${ }^{240}{ }_{94} \mathrm{Pu}$, they have lower capture cross section than the investigated MAs (Table 5). If MAs are present, they absorb relevant part of neutrons and a kind of complicated competition between the isotopes exists. Effectively, it leads to a production of less reactivity, due to the absorbing properties of MAs. A full explanation should take into account detailed spatial effects investigation.

The original BN-600 reactor core fuelled with enriched uranium has six SCR rods with worth equal to $2900 \mathrm{pcm}$ and nineteen SHR rods with $7000 \mathrm{pcm}$, summing up to $9980 \mathrm{pcm}$ for twenty five control rods. Worth to mention that in real $\mathrm{BN}-600$ there are also two additional control rods with $480 \mathrm{pcm}$ - totally $10460 \mathrm{pcm}$ [5]. However, in our model those two rods are not present (Fig. 1), a similar configuration was used in work of Rineiski et al. [22].

In case of the investigated core without MA $(0 \%)$ (driven by plutonium) the total control rods worth is smaller than in the uranium fuelled BN-600 (Table 4) with difference of $1297 \mathrm{pcm}$. It is mainly due to the presence of plutonium isotopes and it is possible to observe that negative effect is further magnified by the addition of MA. In general the addition of transuranium elements reduces control rod worth.

Plutonium and MA have smaller delayed neutrons fractions and those produce more neutrons per one fission than uranium [2]. For example, ${ }^{23}{ }_{92} \mathrm{U}$ produces about 2.45 neutrons per fission for typical sodium fast reactor spectrum and it has delayed neutron fraction equal to about $680 \mathrm{pcm} \mathrm{[2].} \mathrm{On}$ the contrary, the main plutonium isotopes present in the analyzed fuel: (Table 3) ${ }^{239}{ }_{94} \mathrm{Pu}$ generates 2.93 neutrons and $\beta$ equals $215 \mathrm{pcm},{ }^{240}{ }_{94} \mathrm{Pu}$ generates 3.07 neutrons and $\beta$ equals $310 \mathrm{pcm}$ for the same fast spectrum [2]. The most abundant minor actinide ${ }_{241}^{245} \mathrm{Am}$ in case of $1 \mathrm{MeV}$ incident neutron produces 3.37 neutrons and $\beta$ equals about $130 \mathrm{pcm}$ [28].

Whole core delayed neutron fraction is a complicated function of spatial flux, neutron spectra, number of neutrons generated per fission and fission macroscopic cross-sections [2]. Nevertheless, the main source of differences are $\beta$ 's for fuel isotopes, exchanging uranium isotopes into minor actinides will lead to decrease of $\beta$. Similar problem is for effective delayed neutron fraction $\beta_{\text {eff. }}$ In this case additional differences between delayed and prompt neutrons spectra are crucial. Effective delayed neutron fraction is lower than $\beta$ because in typical fast spectrum delayed neutrons have lower probability to cause fission than prompt neutrons for even nuclides [28]. Moreover, minor actinides (mainly americium) have few times higher capture cross-sections for average energies of delayed neutrons in comparison to ${ }^{238}{ }_{92} \mathrm{U}$ [21]. Addition of minor actinides have to decrease delayed neutron fraction and effective delayed neutron fraction. It was confirmed in other studies in case of sodium cooled fast reactors and oxide fuels [21, 28-30].

The considerations presented in a couple of previous paragraphs show reasons of lowered CRW in comparison to real $\mathrm{BN}-600$ for both plutonium fuelled and $\mathrm{Pu}-\mathrm{MA}$ fuelled cores. Thus, it is recommended to perform computations with larger amount of neutron histories to obtain more precise delayed neutron fractions. In order to compare different cores, it is relevant to compare coefficients normalized to effective delayed neutron fractions, because $\beta_{\text {eff }}$ outlines the limit of safe reactor operation but without precise enough $\beta_{e f f}$ it is not possible. 
The reactor core dimension changes due to temperature variation were not taken into account. In fast reactor safety analysis this effects are responsible for insertion of a huge amount of negative reactivity and is believed to be crucial for the overall reactor safety $[2,29]$. For more detailed analysis of reactor safety this issue should be taken into account.

The Doppler constant increases with MA content (it becomes less negative) and it leads to decreasing helpful effect of resonance broadening on the reactor safety - less negative reactivity is inserted into the core in case of fuel heat-up (Fig. 6). Similar conclusion about the core safety can be stated for the sodium void worth. It decreases with MA content and effects in insertion of higher positive reactivity when sodium boils off (Fig. 7). Those are in agreement with further investigations of those reactivity effects which are presented in different works $[2$, $12,17,20,22,28,29,31]$.

\section{Conclusions}

The main purpose of this report was to analyze the safety relevant effects of introduction of minor actinides into a fast reactor core with special emphasis onto effectiveness of the control rods. It was observed that the control rods worth decreases with increasing content of MA, and a possible physical explanation was proposed.

It becomes clear that the addition of minor actinides in order to perform nuclear waste transmutation reduces the effectiveness of the control rods. The obvious recommendation is to increase the control rods fleet in order to maintain similar reactivity safety margin. Nevertheless, the control rods insertion does not provide as fast negative reactivity result as the prompt reactivity effects. Inherent core reactivity feedbacks are principally responsible for minor actinides addition limit in the critical fast reactor cores [20]. This study reveals that control rods effectiveness provides additional obstruction to the addition of minor actinides to the fast reactors fuel.

Acknowledgments. This work is partially based on authors' master thesis. The publication was partially created in the framework of a strategic project NCBR: "Technologies for the development of safe nuclear energy", Research Task No. 9 entitled "Development and implementation of safety analysis methods in nuclear reactors during disturbances in heat removal and severe accident conditions".

\section{References}

1. Bunker, M. E. (1983). Early reactors - from Fermi's water boiler to novel power prototypes. Los Alamos Science, Winter/Spring, 124-131.

2. Waltar, A. E., Reynolds, A., Todd, D. R., \& Tsvetkov, P. V. (2011). Fast spectrum reactors. New York : Springer.

3. Fjaestad, M. (2009, August). Why did the Breed reactor fail? - Swedish and international nuclear develop- ment in a Cold War context. Centre of Excellence for Science and Innovation Studies - Electronic Working Paper Series. Paper No. 186. Stockholm, Sweden. Retrieved November 10, 2013, from: http://www.kth. se/dokument/itm/cesis/CESISWP186.pdf.

4. International Atomic Energy Agency. (2007). Liquid metal cooled reactors: Experience in design and operation. Vienna: Nuclear Power Technology Development Section IAEA. (IAEA-TECDOC-1569).

5. International Atomic Energy Agency. (2006). Fast Reactor Database 2006 Update. Vienna: Nuclear Power Technology Development Section IAEA. (IAEA-TECDOC-1531).

6. U.S. DOE Nuclear Research Advisory Committee and the Generation IV International Forum. (2002). A Technology Roadmap for Generation IV Nuclear Energy Systems.

7. Westlen, D. (2007). Why faster is better - on minor actinide transmutation in hard neutron spectra. Unpublished doctoral dissertation, Royal Institute of Technology, Stockholm, Sweden.

8. Nuclear Energy Agency - Organisation for Economic Co-Operation and Development. (2002). Accelerator-Driven Systems (ADS) and Fast Reactors (FR) in Advanced Nuclear Fuel Cycles - A Comparative Study. Paris: NEA OECD.

9. Westlen, D. (2007). Reducing radiotoxicity in the long run. Prog. Nucl. Energy, 49, 597-605.

10. Salvatores, M., \& Palmiotti, G. (2011). Radioactive waste partitioning and transmutation with advanced fuel cycles: Achievements and challenges. Prog. Part. Nucl. Phys., 66, 144-166.

11. Nifenecker, H., Meplan, O., \& David, S. (2003). Accelerator driven subcritical reactors. Philadelphia, USA: Institute of Physics Publishing.

12. Wallenius, J. (2011). Transmutation of nuclear waste. Royal Institute of Technology. Retrieved August, 2012, from KTH Reactor Physics Division http://neutron. kth.se/courses/Transmutation.shtml.

13. Los Alamos National Laboratory. (2008). MCNP A General Monte Carlo N-Particle Transport Code Version 5. Los Alamos: X-5 Monte Carlo Team.

14. Goorley, T. (2004). Criticality calculations with MCNP5: A primer. Los Alamos: Los Alamos National Laboratory X-5. (LA-UR-04-0294).

15. Darnowski, P. (2013). Neutronic analysis of modified $\mathrm{BN}-600$ fast reactor core with minor actinides. Unpublished master thesis, Warsaw University of Technology, Warsaw, Poland.

16. Aziz, M., \& Hassan, M. I. (2012). Isotopic transmutation and fuel burnup in BN-600 hybrid fast reactor core. Arab J. Nucl. Sci. App., 45(2), 419-426.

17. Grasso, G. (2007). ELSY criticality analysis with MCNP - A preliminary study. Bologna: University of Bologna Nuclear Engineering Laboratory Montecuccolino.

18. Juutilainen, P. (2008). Simulating the behaviour of the fast reactor JOYO. IYNC 2008, 20-26 September 2008 (Paper No. 163). Interlaken, Switzerland.

19. International Atomic Energy Agency. (2010). Hybrid Core Benchmark Analyses Results from a Coordinated Research Project on Updated Codes and Methods to Reduce the Calculational Uncertainties of the LMFR Reactivity Effects. Vienna: Nuclear Power Technology Development Section IAEA. (IAEA-TECDOC-1623).

20. Kim, Y. I., Hill, R., Grimm, K., Newton, T., Li, Z. H., Rineski, A., Mohanakrishan, P., Ishikawa, M., Lee, K. B., Danilytchev, A., \& Stogov, V. (2004). BN-600 Full MOX Core Benchmark Analysis. In PHYSOR 2004 - The Physics of Fuel Cycles and Advanced 
Nuclear Systems: Global Developments, 25-29 April 2004. Chicago, IL, USA: American Nuclear Society.

21. Zhang, Y., Wallenius, J., \& Fokau, Y. (2010). Transmutation of americium in a medium size sodium cooled fast reactor design. Ann. Nucl. Energy, 37, 629-638.

22. Rineiski, A., Ishikawa, M., Jang, J., Mohanakrishnan, P., Newton, T., Rimpault, G., Stanculescu, A., \& Stogov, V. (2011). Reactivity coefficients in BN-600 core with minor actinides. J. Nucl. Sci. Technol., 48, 635-645.

23. Mazgaj, P. E. (2010). Conceptual neutronic design of a $300 \mathrm{MWth}$ lead fast reactor core. Unpublished M.Sc. thesis, Warsaw University of Technology, Warsaw, Poland.

24. Ravnik, M., \& Snoj, L. (2006). Calculation of power density with MCNP in TRIGA reactor. International Conference Nuclear Energy for New Europe, 18-21 September 2006 (Paper No. 109). Portoroz, Slovenia.

25. Michalek, S., Hascik, J., \& Farkas, G. (2008). MCNP5 Delayed Neutron Fraction Calculation in Training Reactor VR-1. J. Electr. Eng., 59, 221-224.
26. Brookhaven National Laboratory. (2013). National Nuclear Data Center. December 10, 2013, from http:// www.nndc.bnl.gov.

27. Lewis, E. E. (2008). Fundamentals of nuclear reactor physics. New York: Academic Press.

28. Wallenius, J. (2012). Physics of americium transmutation. Nucl. Eng. Technol., 44(2), 199-206.

29. Zhang, Y., Wallenius, J., \& Jolkkonen, M. (2013). Transmutation of americium in a large sodium-cooled fast reactor loaded with nitride fuel. Ann. Nucl. Energy, 53, 26-34.

30. Zhang, Y. (2012). Transmutation of Am in sodium fast reactors and accelerator driven systems. Unpublished doctoral dissertation, Royal Institute of Technology, Stockholm, Sweden.

31. Tucek, K., Carlsson, J., \& Wider, H. (2006). Comparison of sodium and lead-cooled fast reactors regarding reactor physics apects severe safety and economical issues. Nucl. Eng. Design, 236, 1589-1598. 